Document downloaded from:

http://hdl.handle.net/10251/70146

This paper must be cited as:

Elena Fito, SF. (2012). RNA virus genetic robustness: possible causes and some consequences. Current Opinion in Virology. 2(5):525-530. doi:10.1016/j.coviro.2012.06.008

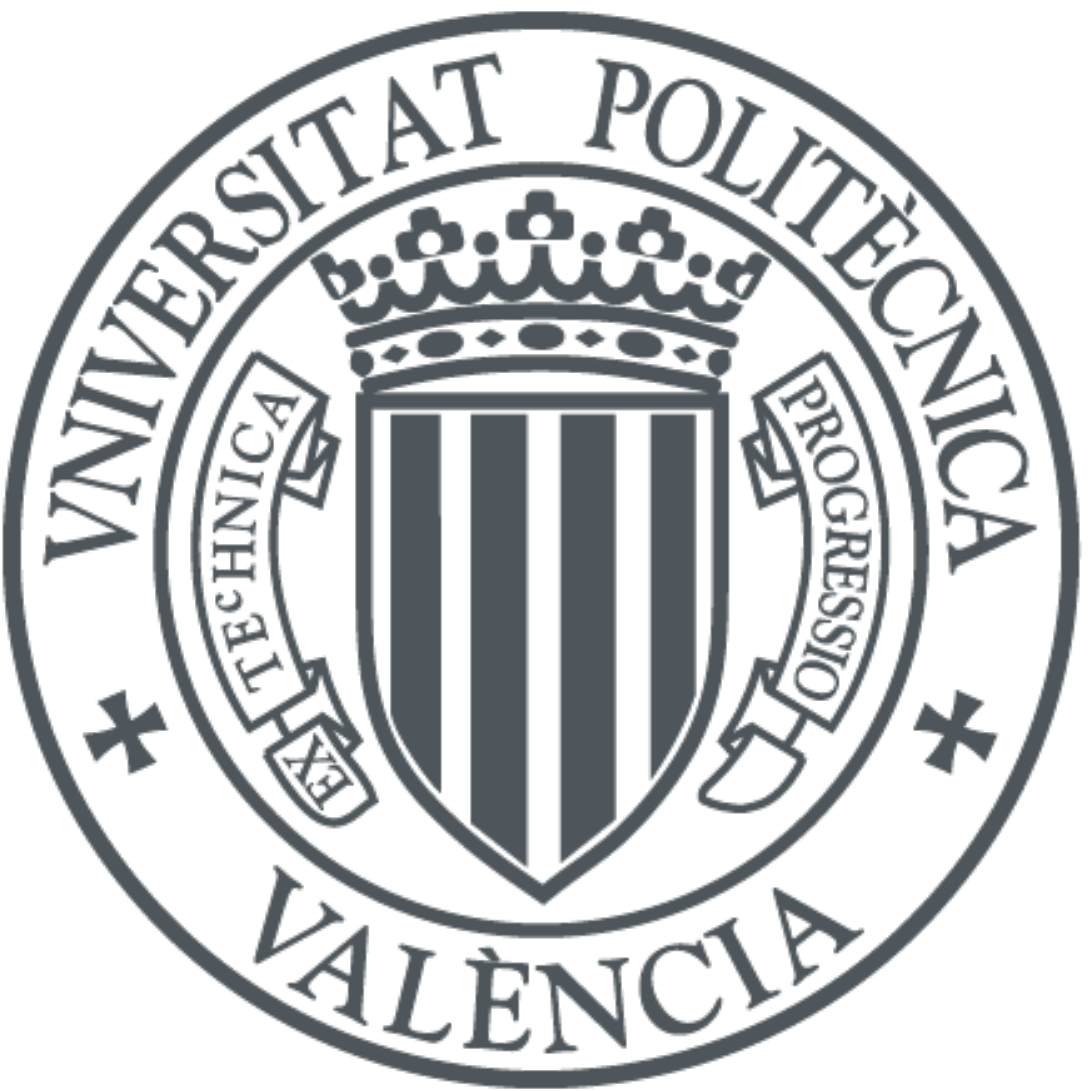

The final publication is available at

http://dx.doi.org/10.1016/j.coviro.2012.06.008

Copyright Elsevier

Additional Information 


\section{RNA Virus Genetic Robustness: Possible Causes and Some Consequences}

Santiago F. Elena ${ }^{1,2}$

${ }^{1}$ Instituto de Biología Molecular y Celular de Plantas, CSIC-UPV, Ingeniero Fausto Elio s/n, 46022 València, Spain

${ }^{2}$ The Santa Fe Institute, 1399 Hyde Road Park, Santa Fe, NM 87501, USA

Contact info: Instituto de Biología Molecular y Celular de Plantas (CSIC-UPV), Campus UPV CPI 8E, Ingeniero Fausto Elio s/n, 46022 València, Spain. Phone: +34 963877 895; Fax: +34 963877 859; E-mail: santiago.elena@csic.es 


\begin{abstract}
In general terms, robustness is the capacity of biological systems to function in spite of genetic or environmental perturbations. The small and compacted genomes and high mutation rates of RNA viruses, as well as the ever-changing environments wherein they replicate, create the conditions for robustness to be advantageous. In this review, I will enumerate possible mechanisms by which viral populations may acquire robustness, distinguishing between mechanisms that are inherent to virus replication and population dynamics and those that result from the interaction with host factors. Then, I will move to review some evidences that RNA virus populations are robust indeed. Finally, I will comment on the implications of robustness for virus evolvability, the emergence of new viruses and the efficiency of lethal mutagenesis as an antiviral strategy.
\end{abstract}

\title{
Highlights
}

- Experimental evidences suggest that viruses may have evolved robustness mechanisms.

- Virus populations are robust at the cost of individuals being fragile.

- Genetic robustness evolves linked to environmental robustness.

- Genetic robustness affects virus evolvability.

- Robustness does not play a substantial role in lethal mutagenesis. 
RNA viruses are the most successful parasites on Earth, infecting hosts from all biological kingdoms, including other parasites. This success results from their evolutionary plasticity (i.e., evolvability): a combination of short generation times, huge population sizes and high mutation rates $[1,2,3]$. Alas, these properties come along with some costs. First, fast replication requires that genomes must be kept small, with overlapping reading frames and encoding multifunctional proteins [4,5]. Second, high mutation rates limit the length of the genome that can be transmitted without incurring in too many errors [6]. High mutation rates may be favored in stressful situations where the input of beneficial mutations allows for escape and survivial (e.g., changing cell types, tissues and hosts or the presence of antiviral responses or drugs). However, in all situations deleterious and lethal mutations represent the larger fraction of all possible mutations [7], thus jeopardizing viral fitness [8,9]. How do RNA viruses maintain their functionality in this scenario? Are they robust to the accumulation of deleterious mutations? In this review I try to answer these questions and look beyond to the consequences of RNA virus robustness.

\section{What is robustness and how can it be measured?}

In a hallmark article, De Visser et al. $\left[10^{* *}\right]$ reviewed the notion of robustness and explored its causes and consequences. Robustness is the preservation of the phenotype in the face of perturbations. The robustness of phenotypes appears at various levels of organization: from gene expression, protein folding, metabolic flux, physiological homeostasis, and development, to fitness. From an evolutionary standpoint, fitness is the most relevant level. Phenotypes can be robust either against mutations or environmental perturbations.

Three reasons may account for the evolution of genetic robustness (GR). First, as long as it is heritable, shows variability among individuals and affects fitness, GR can be a target for selection [11]. The more frequent mutations are, the more efficient selection will be at promoting the evolution of GR. Second, GR is a side effect of stabilizing selection acting on different traits [12]. Third, given that environmental fluctuations often have strong impact on fitness, selection would favor mechanisms of environmental robustness (ER), emerging GR as a correlated response (plastogenetic congruence) $[13,14]$. This is particularly appealing in the case of RNA viruses because 
they must cope not only with deleterious mutations but also with dramatic and fast fluctuations in their environments.

Keeping in mind the definition of GR, a way of estimating it is to evaluate the effect of large collections of individual point mutations on viral fitness. If a point mutation $i$ reduces the fitness of a genotype with respect to that of the wild-type in an amount $s_{i}$, then the average effect $\bar{s}$ across the collection of point mutations can be seen as a measure of mutational sensitivity and, henceforth, as an inverse of GR. In other words, if the average effect of mutations on a virus is small, we conclude it is robust. By contrast, if the average effect is large, we conclude the virus is brittle.

\section{Potential mechanisms for viral GR}

In a previous review, we elaborated on possible mechanisms by which RNA viruses may attain GR $\left[15^{* *}\right]$. We distinguished two classes of mechanisms. Mechanisms of intrinsic GR are the consequence of RNA-genome architecture, replication peculiarities and population dynamics. Intrinsic GR mechanisms operate efficiently at the population level. By contrast, extrinsic GR results from the exploitation of cellular buffering mechanisms by viruses.

This review has been written from the perspective of evolutionary biology. I refer readers interested in molecular details to the excellent review by Barr and Fearns $\left[16^{* *}\right]$ on the several mechanisms by which RNA viruses maintain genome integrity.

\section{Intrinsic mechanisms}

RNA virus genomes are very sensitive to the effect of mutations $\left[7,15^{* *}\right]$, with most mutations being either lethal or strongly deleterious, and with $\bar{s}$ well above $10 \%$ [7]. Furthermore, RNA viruses also show a second hallmark of mutational hypersensitivity, namely, a dominance of antagonistic epistasis among pairs of deleterious mutations $\left[15^{* *}, 17\right]$. Paradoxically, individual hypersensitivity to mutations generates GR at the population level [18]. The efficiency by which natural selection purges deleterious mutations from a population depends on the product $N_{e} \bar{s}$, where $N_{e}$ is the effective population size. RNA viruses reach enormous $N_{e}$ even within infected hosts; hence the above product tends to be large, making selection remarkably efficient removing 
mutants and preserving only non-mutated genomes [18]. In good agreement with this individual hypersensitivity strategy, recent evidences from ultra-deep sequencing of viral populations suggests that much of the variation is rapidly purged from populations and that the wild-type sequence remains numerically dominant, while adaptation to new conditions depends on the fixation of few beneficial alleles $[19,20]$.

Opposite to the individual hypersensitivity strategy is the idea of the survival of the flattest (SF) [21,22] (Figure 1A). When neutral and back mutations are considered, the population average equilibrium fitness depends on the geometry of the fitness landscape, which can be described by $\bar{s}$ [11]. In this scenario, a new selective pressure comes into play at high mutation rates, pushing populations towards regions of the landscape with high density of neutral mutations - a neutral network (NN) (Figure 1B)$[11,21,22]$. As a consequence, the whole population evolves increased GR.

A third mechanism of GR is high ploidy [15,23]. Viruses are $n$-ploid organisms, as $n$ is variable during infection. At initial stages, multiplicity of infection (MOI) is low and viruses are effectively haploid. However, as infection progress, high MOIs ensure frequent co-infections and increasing ploidy. An immediate consequence of polyploidy is genetic complementation. Strong complementation slightly reduces the average population fitness by weakening the efficiency of purifying selection but significantly enhances population diversity and GR, especially if epistasis among deleterious mutations is antagonistic [23].

Different modes of genome replication may also affect GR [24]. By always using the same molecule as template, the stamping-machine strategy produces offspring with a minimal number of mutations, whereas the geometric replication strategy, by using progeny genomes as templates, generates offspring with a number of mutations that increases geometrically. Furthermore, it has been shown that, in combination with selection, the stamping-machine accumulates less mild-effect mutations than geometric replication [24]. Indeed, the difference between both replication schemes in terms of minimizing deleterious mutational load is enhanced if mutations show antagonistic epistasis [24]. 
A last mechanism of intrinsic GR is viral sex, resulting from recombination between homologous molecules or in segregation of segments in a multipartite genome. Sex recreates mutation-free genotypes and helps to keep the average population fitness high. Both forms of sex are common among RNA viruses $\left[15,25^{*}\right]$.

\section{Extrinsic mechanisms}

It is well known that viral infections induce the cellular stress response [26]. However, is it possible that viruses coopt chaperones to buffer mutational effects? The answer is yes. It has been shown that most viruses need cellular chaperones during their life cycle to solve their own protein-folding problems [27], to assist during RNA replication [28] and to interfere with cellular processes such as signal transduction [29].

\section{Evidences of GR in RNA viruses}

The first evidence that RNA viruses have evolved some sort of GR comes from in silico studies analyzing the stability of RNA folding. In a pioneering study, Wagner and Stadler [30] compared the GR of highly conserved RNA secondary structure elements with that of non-conserved elements for three viruses (Denge virus, Hepatitis $C$ virus (HCV) and Human immunodeficiency virus type 1). They hypothesized that conserved elements, given their functional importance must be more robust than non-conserved elements. This hypothesis was supported by the data, thus concluding that the sequences and structures of important conserved domains had evolved to minimize the impact of mutations. Recently, the observation for HCV has been confirmed using a much larger dataset [31].

In a set of computational studies, Sanjuán et al. [32,33] explored the GR of all viroid species. Viroids have been classified into two families according to biological properties and sequence similarity [34]. Interestingly, members of the Avsunviroidae fold into highly branched structures, whilst those from the Pospiviroidae fold into very compact rodlikes. Given that a branched structure seems more fragile than a rodlike, it can be hypothesized that the pospiviroids may show characteristics of GR whereas the avsunviroids may not. Results confirmed this expectation: $\bar{s}$ was much larger for the avsunviroids than for the pospiviroids [32] and epistasis was, on average, antagonistic for the former but synergistic for the later [33]. 
Montville et al. provided the first empirical evidence of evolved GR in an RNA virus $\left[35^{*}\right]$. These authors hypothesized that $\phi 6$ populations evolving at high MOI will experience intense complementation and thus, selection for alternative GR mechanisms will be weak. By contrast, populations evolved at low MOI will evolve alterative GR mechanisms. After 300 generations of experimental evolution, clones from each of three independent evolution lineages per treatment were isolated and subjected to 100 generations of mutation-accumulation (MA) by genetic drift at low MOI $\left[35^{*}\right]$. If the initial hypothesis was true, then viruses evolved at high MOI will show no GR and will experience larger fitness declines than those evolved at low MOI. The results significantly matched this expectation, thus confirming that GR could evolve in $\phi 6$ after just 300 generations.

Confirmation of the SF effect as a mechanism of GR came from two different experiments. Codoñer et al. [36] selected two different viroids that infected a common host. These two viroids largely differ in their replication rates and in the extent of genetic variability they generated within host. Chrysanthemum chlorotic mottle viroid (CChMVd) generated lots of variants after being inoculated but accumulated to very low titers. Chrysanthemum stunt viroid (CSVd) accumulated to very high titers but showed little genetic variation. The authors hypothesized that CChMVd may represent a case of a flat organism replicating in a $\mathrm{NN}$ whereas CSVd may not so. To test this hypothesis both viroids were co-inoculated into the same plants and allowed to compete. As expected, CSVd quickly outcompeted CChMVd owed to its faster replication rate (Figure 1A). However, when mutation rate was artificially increased by UVC radiation, the situation was reversed and CChMVd persisted in the mixed population (Figure 1A).

Sanjuán et al. [37] provided a second confirmation of the SF effect. Two Vesicular stomatitis virus (VSV) populations that differed in evolutionary history were chosen. Population A was formed by individuals that on average had lower fitness than those from population B but that were more diverse in fitness (Figure 1A). The authors hypothesized that population A was the flattest while population B was the fittest. As in the viroids case, these two populations were allowed to compete in standard conditions and at increasing mutation rates (by adding either 5-FU or 5-AzC). The results showed 
that while population B outcompeted population A at standard conditions, B was able of reverse its fortune as the concentration of mutagens increased.

As I mentioned at the beginning of this review, it has been proposed that plastogenetic congruence may drive the evolution of GR. To test this hypothesis Domingo-Calap et al. $\left[38^{*}\right]$ evolved independent populations of $\mathrm{Q} \beta$ under periodic temperature pulses to select for thermotolerant viruses. Thermotolerant and control viruses were then subjected to MA by treating populations with $\mathrm{HNO}_{2}$ at each experimental passage. If selection for ER has a positive effect on GR, then thermotolerant viruses may suffer a smaller reduction in fitness than the control viruses during the MA phase. The results confirmed this expectation, thus supporting the view that GR evolves as a correlated response to selection for ER.

\section{Consequences of GR}

\section{Does GR promote evolvability?}

There is not easy answer to this question since opposing results have been reported.

McBride et al. [39] used some of the $\phi 6$ robust and brittle clones generated in [35 $]$ to test whether they differed in their ability to adapt to a new thermal niche. All clones were originally evolved at $25^{\circ} \mathrm{C}$ and had very low viability above $45^{\circ} \mathrm{C}$. The selected clones were evolved for 50 generations under periodic pulses at $45^{\circ} \mathrm{C}$. At the end of this evolution phase, the fitness of all evolved lines was tested at $45{ }^{\circ} \mathrm{C}$. As expected, the robust clones had achieved higher fitness than the brittle ones.

The existence of NN has a strong implication for the antigenic evolution of Influenza A virus $\mathrm{H} 3 \mathrm{~N} 2\left[40,41^{*}\right]$. The observed patterns of epochal antigenic evolution of H3N2, alternating periods of phenotypic stasis punctuated by sudden changes in the antigenic phenotype [40] can easily be explained in terms of NN [41 ${ }^{*}$ (Figure 1B). At the onset of an epochal evolution cycle, a $\mathrm{H} 3 \mathrm{~N} 2$ population is distributed over the $\mathrm{NN}$ of an antigenic cluster (Figure 1B). Neutral mutations accumulate, allowing the virus to explore and reach distant regions of the NN. At some point, a mutation in the edge of the network will create an individual that belongs to a new NN that corresponds to a different antigenic cluster (Figure 1B). This antigenic innovation corresponds to a peak 
in infections. The new antigenic variant now starts exploring the new $\mathrm{NN}$, and the process repeats itself.

Turner et al. [42] have tested whether generalist (i.e., environmentally robust) viruses were more evolvable than specialist (i.e., environmentally brittle) ones. To do so, they used VSV populations that were previously evolved as generalists or as specialists. The fitness of all these populations was tested on four novel hosts. The prediction was that the ER generalists would show higher mean fitness and less variance in mean fitness across the novel hosts than the brittle specialists. These predictions were fulfilled, thus linking robustness to the likelihood of viral emergence.

Contrasting to the above results, Cuevas et al. [43] have shown that brittle VSV populations were more evolvable than genetically robust ones when facing a new host cell type. Brittle populations reached higher infectivity and fitness in the new cell line than the robust ones, while both paid the same fitness cost in the ancestral host cell type and accumulated similar number of mutations.

Two arguments can be brought forward to explain this discrepancy. First, the relationship between robustness and evolvability may be time-dependent $[44,45]$. At the short term GR will buffer the effect of any potential beneficial mutation, thus hampering adaptation. Only at the long-term will GR bolster evolvability by allowing populations to drift in the NN until reaching distant parts and facility the switch to different NN. Second, to confer GR and evolvability, the size of the NN needs to cover most of the genotypic space; otherwise only small regions would be explored [46].

New data need to be obtained to solve this controversy.

\section{Does GR diminish lethal mutagenesis?}

Lethal mutagenesis (LM), that is viral extinction mediated by enhanced mutation rates, has been proposed as a potential therapeutic strategy $\left[47^{*}\right]$. A critical issue regarding LM as an antiviral strategy is whether virus mutants resistant to the mutagens can be

selected. Obviously, GR may be relevant for the emergence of such mutants. The scarce data available do not provide a definitive answer to the above question. 
To tackle the problem of whether passages in presence of mutagens may select for genetically robust genomes that will jeopardize the efficiency of LM, Martín et al. [48] evolved populations of LCMV in absence and in presence of a sub-lethal concentration of 5-FU. Populations and clones isolated at different time points during the evolution experiment were then tested for their resistance to a concentration of 5-FU large enough as to induce LM. No differences in the outcome of the experiment were observed between viruses evolved in presence or absence of 5-FU. This observation led to the conclusion that evolution in presence of sub-lethal concentrations of mutagen did not select for GR.

Recently, Graci et al. [49] have provided evidences that GR determines the success of LM. These authors have shown that the enteroviruses Coxsackie virus B3 (CVB3) and Polio virus (PV) differ in their degree of GR according to a series of evidences, the former being less robust than the latter. In agreement with the hypothesis that GR will diminish the efficiency of LM, the results showed that CVB3 was more sensitive to ribavirin that was PV.

However, a theoretical analysis of the effect or GR on the likelihood of extinction by LM [50] has shown that the effect of GR on LM shall be minor. Viruses will obtain a benefit from GR only if the increase in mutation rate by the mutagen is small. When the mutation rate goes beyond a critical value that depends on the ratio between the logarithm of the virus reproductive capacity and the fraction of all deleterious mutations, the virus will not have time enough as to expand its NN. The study concludes that GR does not impose a strong burden to LM therapy.

\section{Concluding remarks}

Far from being passive victims of their error-prone replication, RNA viruses cope with the deleterious effects of mutations. Growing evidences suggest that viruses have evolved mechanisms to increase their GR at the population level at the cost of being very fragile at the individual level. The role of GR in the emergence of new viruses or in the durability of antiviral therapies needs to be further explored.

\section{Acknowledgements}


This work was supported by the Spanish MICINN grant BFU2009-06993 and by the Santa Fe Institute. I thank Mark P. Zwart for critical reading of the manuscript.

\section{References and recommended reading}

Papers of particular interest, published within the period of review, have been highlighted as:

* of special interest

** of outstanding interest

1. Domingo E: Viruses at the edge of adaptation. Virology 2000, 270:251-253.

2. Elena SF, Sanjuán R: Virus evolution: insights from an experimental approach. Annu. Rev. Ecol. Evol. Syst. 2007, 38:27-52.

3. Holmes EC: The evolutionary genetics of emerging viruses. Annu. Rev. Ecol. Evol. Syst. 2009, 40:353-372.

4. Belshaw R, Pybus OG, Rambaut A: The evolution of genome compression and genomic novelty in RNA viruses. Genome Res. 2007, 17:1496-1504.

5. Chirico N, Vianelli A, Belshaw R: Why genes overlap in viruses. Proc. R. Soc. B 2010, 277:3809-3817.

6. Holmes EC: Error threshold and the constraints to RNA virus evolution. Trends Microbiol. 2003, 11:543-546.

7. Sanjuán R: Mutational fitness effects in RNA and single-stranded DNA viruses: common patterns revealed by site-directed mutagenesis studies. Phil. Trans. $R$. Soc. B 2010, 365:1975-1982.

8. Elena SF, Sanjuán R: Adaptive value of high mutation rates of RNA viruses: separating causes from consequences. J. Virol. 2005, 79:11555-11558.

9. Belshaw R, Gardner A, Rambaut A, Pybus OG: Pacing a small cage: mutation and RNA viruses. Trends Ecol. Evol. 2007, 23:188-193.

10. ** De Visser JAGM, Hermisson J, Wagner GP, Ancel Meyers L, BagheriChaichian H, Blanchard JL, Chao L, Cheverud JM, Elena SF, Fontana W et al:: Perspective: evolution and detection of genetic robustness. Evolution 2003, 57:1959-1972.

This perspective article represents a landmark in the research about genetic robustness. It reviewed previous notions from different fields, settled the definitions and revitalized the interest of evolutionary biologists on the topic. 
11. Wilke CO, Adami C: Evolution of mutational robustness. Mut. Res. 2003, 522:311.

12. Meiklejohn CD, Hartl DL: A single mode of canalization. Trends Ecol. Evol. 2002, 17:468-473.

13. Ancel LW, Fontana W: Plasticity, evolvability and modularity in RNA. J. Exp. Zool. 2000, 288:242-283.

14. Wagner WP, Booth $\mathrm{G}$, Bagheri-Chaichian $\mathrm{H}$ : A population genetic theory of canalization. Evolution 1997, 51:329-347.

15. ** Elena SF, Carrasco P, Daròs JA, Sanjuán R: Mechanisms of genetic robustness in RNA viruses. EMBO Reports 2006, 7:168-173.

This is the first thoughtful review of the possible mechanisms that may generate robustness in viral populations.

16. ** Barr JN, Fearns R: How RNA viruses maintain their genome integrity. $J$. Gen. Virol. 2010, 91:1373-1387.

In this timely review article, readers will find molecular mechanisms by which RNA viruses correct mistakes produced during replication as well as protect and repair their extremes.

17. Elena SF, Solé RV, Sardanyés J: Simple genomes, complex interactions: epistasis in RNA virus. Chaos 2010, 20:026106.

18. Krakauer DC, Plotkin JB: Redundancy, antiredundancy and the robustness of genomes. Proc. Natl. Acad. Sci. USA 2002, 99: 1405-1409.

19. Wright CF, Morelli M, Thébaud G, Knowles NJ, Herzyk P, Paton DJ, Haydon DT, King DP: Beyond the consensus: dissecting within-host viral population diversity of Foot-and-mouth disease virus by using next-generation genome sequencing. $J$. Virol. 2011, 85:2266-2275.

20. Martínez F, Lafforgue G, Morelli MJ, González-Candelas F, Chua NH, Daròs JA, Elena SF: Ultra-deep sequencing analysis of population dynamics of virus escape mutants in RNAi-mediated resistant plants. Mol. Biol. Evol. 2012, doi: $10.1093 / \mathrm{molbev} / \mathrm{mss} 135$.

21. Schuster P, Swetina J: Stationary mutant distributions and evolutionary optimization. Bull. Math. Biol. 1988, 50:635-660.

22. Wilke CO, Wang JL, Ofria C, Lenski RE, Adami C: Evolution of digital organisms at high mutation rates leads to survival of the flattest. Nature 2001, 412:331-333. 
23. Gao H, Feldman MW: Complementation and epistasis in viral coinfection dynamics. Genetics 2009, 182:251-263.

24. Sardanyés J, Solé RV, Elena SF: Replication mode and landscape topology differentially affect RNA virus mutational load and robustness. J. Virol. 2009, 23:12579-12589.

25. * Simon-Loriere E, Holmes EC: Why do RNA viruses recombine? Nat. Rev. Microbiol. 2011, 9:617-626.

This is a recommendable reading for those interested in the effect of recombination in virus evolution. Especially for those that still believe recombination must be beneficial perse.

26. Whitham SA, Yang C, Goodin MM: Global impact: elucidating plant responses to viral infection. Mol. Plant-Microb. Interact. 2006, 11:1207-1215.

27. Jockusch H, Wiegand C, Mersch B, Rajes D: Mutants of Tobacco mosaic virus with temperature-sensitive coat proteins induce heat shock response in tobacco leaves. Mol. Plant-Microb. Interact. 2001, 7:914-917.

28. Zúñiga S, Sola I, Cruz JLG, Enjuanes L: Role of RNA chaperones in virus replication. Virus Res. 2009, 139:253-266.

29. Mayer MP: Recruitment of Hsp70 chaperones: a crucial part of viral survival strategies. Rev. Physiol. Biochem Pharmacol. 2005, 153:1-46.

30. Wagner A, Stadler PF: Viral RNA and evolved mutational robustness. J. Exp. Zool. 1999, 285:119-127

31. Churkin A, Cohen M, Shemer-Avni Y, Barash D: Bioinformatic analysis of the neutrality of RNA secondary structure elements across genotypes reveals evidence for direct evolution of genetic robustness in HCV. J. Bioinform. Comput. Biol. 2010, 8:1013-1026.

32. Sanjuán R, Forment J, Elena SF: In silico predicted robustness of viroid RNA secondary structures. I. The effect of single mutations. Mol. Biol. Evol. 2006, 23: $1427-1436$.

33. Sanjuán R, Forment J, Elena SF: In silico predicted robustness of viroid RNA secondary structures. II. Interactions between mutation pairs. Mol. Biol. Evol. 2006, 23:2123-2130.

34. Elena SF, Dopazo J, Flores R, Diener TO, Moya A: Phylogeny of viroids, viroidlike satellite RNAs, and the viroidlike domain of hepatitis $\delta$ virus. Proc. Natl. Acad. Sci. USA 1991, 88:5631-5634. 
35. * Montville R, Froissart R, Remold SK, Tenaillon O, Turner PE: Evolution of mutational robustness in an RNA virus. PLoS Biol. 2005, 3:e281.

First experimental demonstration that RNA viruses can evolve mutational robustness.

36. Codoñer FM, Daròs JA, Solé RV, Elena SF: The fittest versus the flattest: experimental confirmation of the quasispecies effect with subviral pathogens. PLoS Pathog. 2006, 2:e136.

37. Sanjuán R, Cuevas JM, Furió V, Holmes EC, Moya A: Selection for robustness in mutagenized RNA viruses. PLoS Genet. 2007, 3:e93.

38. * Domingo-Calap P, Pereira-Gómez M, Sanjuán R: Selection for thermostability can lead to the emergence of mutational robustness in an RNA virus. J. Evol. Biol. 2010, 23:2453-2460.

First experimental demonstration of the plastogenetic congruence hypothesis: mutational robustness evolves in RNA viruses as a side effect of selection for environmental robustness.

39. McBride RC, Ogbunugafor $\mathrm{CB}$, Turner PE: Robustness promotes evolvability of thermotolerance in an RNA virus. BMC Evol. Biol. 2008, 8:231.

40. Koelle K, Cobey S, Grenfell BT, Pascual M: Epochal evolution shapes the phylodynamics of interpandemic influenza A (H3N2) in humans. Science 2006, 314:1898-1903.

41. *Van Nimwegen E: Influenza escapes immunity along neutral networks. Science 2006, 314:1884-1886.

Nice description of the effect of neutral networks in the ability of influenza A virus to explore a neutral genotypic space and reach solutions to escape from immune pressure.

42. Turner PE, Morales NM, Alto BW, Remold SK: Role of evolved host breadth in the initial emergence of an RNA virus. Evolution 2010, 64:3273-3286.

43. Cuevas JM, Moya A, Sanjuán R: A genetic background with low mutational robustness is associated with increased adaptability to a novel host in an RNA virus. J. Evol. Biol. 2009, 22:2041-2048.

44. Elena SF, Sanjuán R: The effect of genetic robustness on evolvability in digital organisms. BMC Evol. Biol. 2008, 8:284.

45. Wagner A: Neutralism and selectionism: a network-based reconciliation. Nat. Rev. Genet. 2008, 9:965-974.

46. Ciliberti $\mathrm{S}$, Martin $\mathrm{OC}$, Wagner $\mathrm{A}$ : Innovation and robustness in complex regulatory gene networks. Proc. Natl. Acad. Sci. USA 2007, 104:13591-13596. 
47. * Ruíz-Jarabo CM, Ly C, Domingo E, de la Torre JC: Lethal mutagenesis of the prototypic arenavirus Lymphocytic choriomeningitis virus (LCMV). Virology 2003, 308: 37-47.

First experimental evidence that lethal mutagenesis may work as an antiviral therapy in vivo.

48. Martín V, Grande-Pérez A, Domingo E: No evidence of selection for mutational robustness during lethal mutagenesis of Lymphocytic choriomeningitis virus. Virology 2008, 378:185-192.

49. Graci JD, Gnädig NF, Galarraga JE, Castro C, Vignuzzi M, Cameron CE: Mutational robustness of an RNA virus influences sensitivity to lethal mutagenesis. J. Virol. 2012, 86:2869-2873.

50. O'Dea EB, Keller TE, Wilke CO: Does mutational robustness inhibit extinction by lethal mutagenesis in viral populations? PLoS Comput. Biol. 2010, 6:e1000811. 
Figure 1. (A) Schematic representation of the survival of the flattest effect. In this twodimensional representation of fitness landscapes, fitness corresponds to the height on the peaks, whereas the horizontal axis represents different genotypes. Fast replicating but brittle populations inhabit the left peak, whereas slow replicating yet robust populations inhabit the right peak. At low mutation rates (upper panel), populations remain located at their peaks and the fittest (brittle) outcompetes the flattest (robust). At high mutation rates (lower panel), mutations move genotypes away from their original position on the peaks. The fittest viral population experiences large changes in fitness, as genotypes slide down from the narrow fitness peak. Because the flattest population inhabits a neutral portion of the landscape, its fitness is buffered against mutational change. (Modified from ref. [37]. Reprinted with permission from the authors.) (B) Influenza A virus H3N2 antigenic evolution in a neutral network model. Phenotypes are genetically robust, allowing genotypes to drift through genotypic neutral space until reaching the edge of a new neutral network (that corresponds to a different antigenic variant) before instant shifts in phenotype. (Modified from Ref. [41]. Reprinted with permission from AAAS.) 
(A)
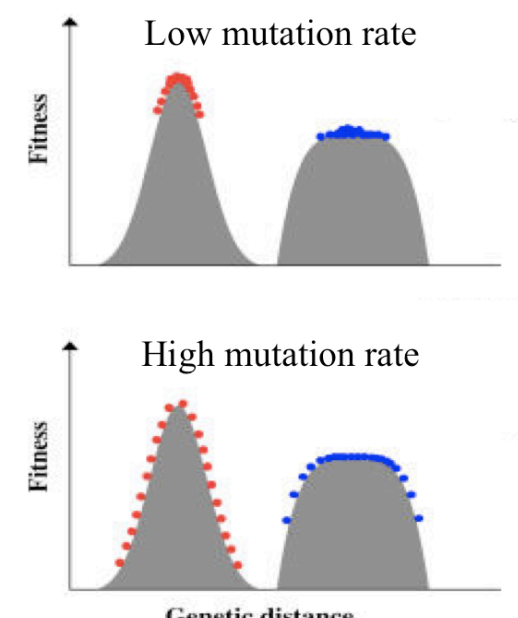

(B)

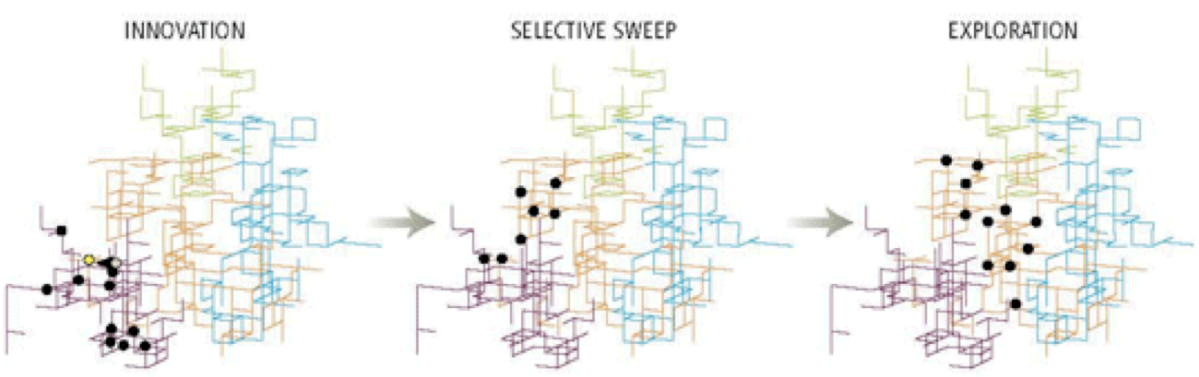

